\title{
Differentiation of 1-O-alk-1'-enyl-2-acyl and 1-O-alkyl-2-acyl Glycerophospholipids by Multiple-Stage Linear Ion-Trap Mass Spectrometry with Electrospray Ionization
}

\author{
Fong-Fu Hsu and John Turk \\ Mass Spectrometry Resource, Division of Endocrinology, Diabetes, Metabolism, and Lipid Research, \\ Department of Internal Medicine, Washington University School of Medicine, St. Louis, Missouri, USA
}

\begin{abstract}
We described linear ion-trap mass spectrometric approaches applying $\mathrm{MS}^{3}$ and $\mathrm{MS}^{4}$ toward to the structural characterization of 1-O-alk-1'-enyl-2-acyl-, 1-O-alkyl-2-acyl-, and diacylglycerophospholipids (GPL) as the $[\mathrm{M}-\mathrm{H}]^{-}$ions desorbed by ESI in negative-ion mode. Further dissociation of the $\left[1 \mathrm{M}-\mathrm{H}-\mathrm{R}_{2} \mathrm{CO}_{2} \mathrm{H}-\text { polar head group }\right]^{-}$ions from the $[\mathrm{M}-\mathrm{H}]^{-}$ions of GPL that have undergone the consecutive losses of the fatty acid substituent at $s n-2$ and the polar head group readily gives the structural information of the radyl group at $s n-1$, resulting in structural differentiation among the 1-O-alk-1'-enyl-2-acyl-, 1-O-alkyl-2-acyl, and diacylglycerolphospholipid molecules. The distinction between a 1-O-alk-1'-enyl-2-acyl- and a 1-O-alkyl-2-acyl-GPL is based on the findings that the $\mathrm{MS}^{3}$ (or MS ${ }^{4}$ ) spectrum of the $[\mathrm{M}-\mathrm{H}-$ $\mathrm{R}_{2} \mathrm{CO}_{2} \mathrm{H}$ - polar head group $]^{-}$ion from the former compound is dominated by the alkenoxide anion that represents the radyl moiety at $s n-1$, while the spectrum from the latter compound is dominated by the ion at $\mathrm{m} / \mathrm{z} 135$ arising from further loss of the 1-O-alkyl group as an alcohol. Another important notion is that the optimal collision energy required for acquiring the former spectrum is significantly lower than that required for obtaining the latter spectrum. Using the approaches, we are able to reveal the structures of several isobaric isomers in GPL mixtures of biological origin. Because the $[\mathrm{M}-\mathrm{H}]^{-}$ions are readily formed by various GPL classes (except glycerophosphocholine) in the negative-ion mode, these mass spectrometric approaches should have broad application in the structural identification of GPLs. (J Am Soc Mass Spectrom 2007, 18, 2065-2073) @ 2007 American Society for Mass Spectrometry
\end{abstract}

$\mathrm{T}$ The subclasses of plasmanyl and plasmenyl lipids consist of a 1-O-alkyl and a 1-O-alk-1'-enyl group bonded to the $s n-1$ position of the glycerol backbone, respectively; while a fatty acyl group is attached to the $s n-2$ position of the glycerol backbone by an ester bond. The differentiation of a plasmenyl glycerophospholipid (GPL) (plasmalogen) from a plasmanyl GPL by tandem mass spectrometry has been a difficult task due to the structural similarity between the two classes of compounds. Several mass spectrometric approaches have been employed. For examples, Murphy et al. described the confirmation of the plasmalogen glycerophosphoethanolamine (GPEtn) (plasmenylethanolamine) molecules in lipid mixture by comparison of the mass spectra of the lipid mixtures before and after the removal of plasmalogen GPEtn by acid treatment $[1,2]$. The approach is simple but destructive and resulted in a severe sample loss [3]. Hsu and Turk described tandem quadrupole mass spectrometric method to characterize plasmenyl- and plasmanyl glycerophos-

Address reprint requests to Dr. F.-F. Hsu, Box 8127, Washington University School of Medicine, 660 S. Euclid, St. Louis, MO 63110, USA. E-mail: fhsu@im.wustl.edu phocholines (GPCho) using their lithiated ions desorbed by ESI, the structures of both the plasmenyl- and plasmanyl GPCho, including the identities of the radyl groups at the glycerol backbone and the polar head group can be obtained [4]. The product-ion spectra of the $[\mathrm{M}+\mathrm{Li}]^{+}$ion of plasmalogen GPEtn after collisionally activated dissociation (CAD) also contain the fragment ions that readily reflect the radyl groups, leading to unambiguous structural assignment of the structure [5]. Similar results were also reported by Berry and Murphy, who described the structural identification of plasmalogen GPEtn species as the $[\mathrm{M}+\mathrm{H}]^{+}$ion desorbed by ESI, followed by multiple-stage IT mass spectrometry [2]. The prominent fragment ions from $\mathrm{MS}^{3}$ that reflect the fatty acyl substituent at $s n-2$ (seen as a $\left[\mathrm{R}_{2} \mathrm{CO}_{2} \mathrm{H}+56+\mathrm{H}\right]^{+}$ion), and the 1-O-alk-1'-enyl moiety at $s n-1$ (seen as a $\left[\mathrm{M}+\mathrm{H}-\mathrm{R}_{2} \mathrm{CO}_{2} \mathrm{H}-56\right]^{+}$ion) afford unambiguous assignment of plasmalogen GPEtn structures [2].

While the $[\mathrm{M}+\mathrm{Li}]^{+}$and $[\mathrm{M}+\mathrm{H}]^{+}$ions are readily formed by the GPCho and GPEtn classes upon ESI in positive-ion mode, phospholipid classes of GPEtn, glycerophosphatidic acid (GPA), glycerophosphoinositol (GPIns), glycerophosphoserine (GPSer), and glycero- 
phosphoglycerol (GPGro) yield abundant ions in the $[\mathrm{M}-\mathrm{H}]^{-}$form in negative-ion mode. The CAD production spectra of the $[\mathrm{M}-\mathrm{H}]^{-}$ions from diacyl glycerophospholipids have been routinely used for their structural characterization, including the assignments of the fatty acid substituents at the glycerol backbone [6, 7]. By contrast, the $\mathrm{MS}^{2}$ spectra of the $[\mathrm{M}-\mathrm{H}]^{-}$ions from both a 1-O-alkyl-2-acyl and a 1-O-alk-1'-enyl-2-acyl glycerolphospholipids obtained with a triple-stage quadrupole (TSQ) or an ion-trap instrument only give the carboxylate anion at $s n$-2 (i.e., the $\mathrm{R}_{2} \mathrm{CO}_{2}{ }^{-}$ion) and of the ions arising from losses of the fatty acid substituent at $s n-2$ as an acid and as a ketene (i.e., the $\left[\mathrm{M}-\mathrm{H}-\mathrm{R}_{2} \mathrm{CO}_{2} \mathrm{H}\right]^{-}$and $\left[\mathrm{M}-\mathrm{H}-\mathrm{R}_{2} \mathrm{CH}=\mathrm{CO}\right]^{-}$ions), respectively. These ions are informative for determination of the fatty acyl group at $s n-2$, but the structural information regarding to the identity of the radyl group at $s n-1$ is not available. Thus, differentiation of a plasmanyl GPL from a plasmenyl GPL by their $\mathrm{MS}^{2}$ product-ion spectra from the $[\mathrm{M}-\mathrm{H}]^{-}$ions is not readily applicable.

Herein, we described the multiple-stage ion-trap mass spectrometric approaches employing $\operatorname{MS}^{\mathrm{n}}(n=3$, 4) for characterization of plasmenyl- and plasmanylGPLs. The distinction between the $\operatorname{MS}^{n}(n=3,4)$ spectra from a 1-O-alkyl-2-acyl GPL and a 1-O-alk-1'enyl-2-acyl GPL afforded unambiguous differentiation of a plasmenyl-GPL from a plasmanyl-GPL.

\section{Materials}

All chemicals used are in spectroscopic grade and were purchased from Sigma Chemical Co (St. Louis, $\mathrm{MO})$. The 1-O-octadec-1'-enyl-2-oleoyl-sn-glycero-3phosphoethanolamine was purchased from Avanti Polar Lipid Co. (Alabaster, Al), while the 1-O-octadecanyl-2steroyl-sn-glycero-3-phosphoethanolamine was prepared by hydrogenation of 1-O-octadec-1'-enyl-2-oleoyl-snglycero-3-phosphoethanolamine. The lipid extracts from Leishmania major (L. major) and African trypanosomes were prepared as previously described $[8,9]$.

\section{Methods}

\section{Mass Spectrometry}

Low-energy CAD tandem mass spectrometry experiments were conducted on a Finnigan (San Jose, CA) linear ion-trap (LIT) mass spectrometer with Xcalibur operating system. Accurate mass measurements were performed on a Finnigan LIT-FT instrument. Lipid solution in methanol ( 1 to $5 \mathrm{pmol} / \mu \mathrm{L}$ ) was infused (2 $\mu \mathrm{L} / \mathrm{min}$ ) to the ESI source, where the skimmer was set at ground potential, the electrospray needle was set at $4.5 \mathrm{kV}$, and temperature of the heated capillary was $300{ }^{\circ} \mathrm{C}$. The automatic gain control of the ion trap was set to $5 \times 10^{4}$, with a maximum injection time of $400 \mathrm{~ms}$. Helium was used as the buffer and collision gas at a pressure of $1 \times 10^{-3}$ mbar ( 0.75 mTorr). The $\mathrm{MS}^{n}$ experiments were carried out with an optimized rela- tive collision energy ranging from $16 \%$ to $20 \%$ and with an activation q value at 0.25 , and the activation time at 30 to $50 \mathrm{~ms}$ to leave a minimal residual abundance of precursor ion (around 20\%). Mass spectra were accumulated in the profile mode, typically for 3 to $5 \mathrm{~min}$ for $\mathrm{MS}^{2}$ - and $\mathrm{MS}^{3}$ spectra. The mass resolution of the instrument was tuned to 0.6 Da at half peak height.

\section{Nomenclature}

The abbreviations previously described for plasmanyl and plasmenyl (plasmalogen) lipids were used. The 1-O-alk-1'-enyl-2-acyl glycerophospholipids (plasmalogens), for example, the 1-O-octadec-1'-enyl-2-oleoylsn-glycero-3-phosphoethanolamine was designated as p18:0/18:1-GPEtn; while the 1-O-alkyl-2-acyl glycerophospholipids (plasmanyl lipids), for example, the 1-Ooctadecanyl-2-steroyl-sn-glycero-3-phosphoethanolamine was designated as a18:0/18:0-GPEtn. In text, we also used, for example, plasmenyl glycerophospholipid (plasmenyl-GPL) to signify the plasmalogens with the understanding that "plasmenyl" bears 1-O-alk-1'-enyl2-acyl of sn-glycero-3-phosphate. The product-ion spectrum from $\mathrm{MS}^{\mathrm{n}}(n=2,3, \ldots)$ experiments is designated as $\mathrm{MS}^{\mathrm{n}}$ spectrum.

\section{Results and Discussion}

\section{Characterization of Plasmenyl and Plasmanyl Glycerophosphoethanolamines}

The $\mathrm{MS}^{2}$ product-ion spectrum of the $[\mathrm{M}-\mathrm{H}]^{-}$ion of a plasmanyl or a plasmenyl- GPL obtained by a tandem quadrupole or an ion-trap instrument contains limited structural information that only the fatty acyl substituent at $s n-2$ can be determined. For example, the LIT MS ${ }^{2}$ spectrum of the $[\mathrm{M}-\mathrm{H}]^{-}$ion of $p 18: 1 / 18: 1-G P E t n$ at $\mathrm{m} / \mathrm{z} 726$ (Figure 1a) contains a prominent ion at $\mathrm{m} / \mathrm{z} 281$, corresponding to a 18:1-carboxylate anion, along with the ions at $\mathrm{m} / \mathrm{z} 462$ and 444, arising from losses of the 18:1-fatty acyl substituent at $s n-2$ as a ketene and as an acid, respectively. These ions reflect the 18:1-fatty acid substituent at $s n-2$, and the ions reflecting the radyl group at $s n-1$ are not available. The feature ions at $\mathrm{m} / \mathrm{z}$ 140 and 196 that are commonly seen for a GPEtn [10] are also absent due to the low-mass cutoff of an ion-trap instrument. These two ions, however, are readily seen in the tandem quadrupole mass spectrum of $\mathrm{m} / \mathrm{z} 726$ (data not shown) [10]. Similarly, the LIT MS ${ }^{2}$ spectrum of the $[\mathrm{M}-\mathrm{H}]^{-}$ion of a18:0/18:0-GPEtn at $\mathrm{m} / \mathrm{z} 732$ (Figure 1d) contains a major 18:0-carboxylate anion at $\mathrm{m} / \mathrm{z} 283$, along with the ions at $\mathrm{m} / \mathrm{z} 466$ and 448, corresponding to losses of the 18:0-fatty acyl substituent at $s n-2$ as a ketene and as an acid, respectively. The profiles of the two spectra (Figure 1a and d) are nearly identical, indicating that differentiation of a plasmenylethanolamine from a plasmanylethanolamine by their $\mathrm{MS}^{2}$ spectra is not possible. 

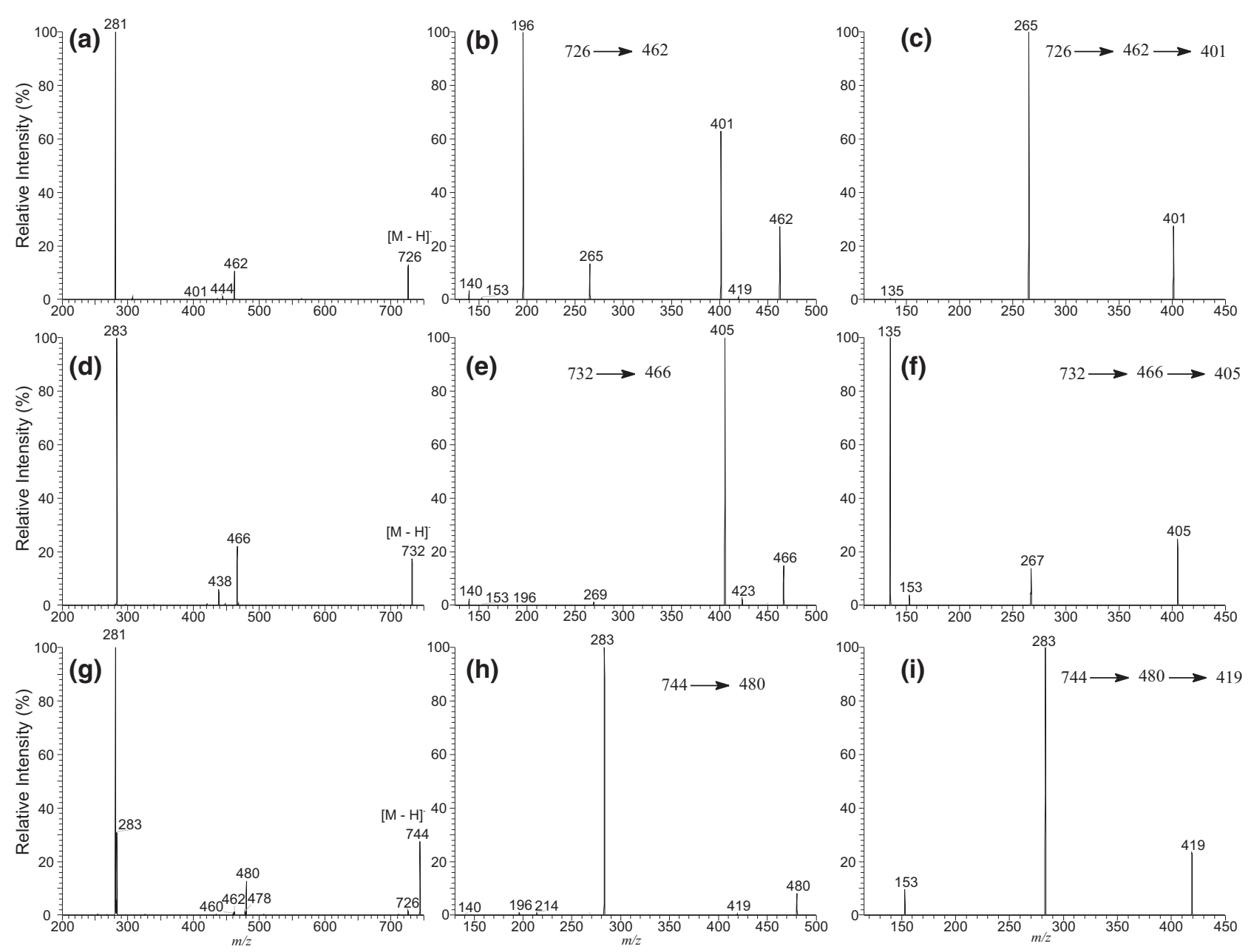

Figure 1. The LIT MS ${ }^{2}$ spectra of the $[\mathrm{M}-\mathrm{H}]^{-}$ions of $p 18: 1 / 18: 1$-GPEtn at $\mathrm{m} / \mathrm{z} 726$ (a), of a18:0/18:0-GPEtn at $m / z 732(\mathbf{d})$, of 18:0/18:1- GPEtn at $m / z 744(\mathrm{~g})$, their $\mathrm{MS}^{3}$ spectra of the ions at $m / z 462(726 \rightarrow 462)($ b) , at $m / z 466(732 \rightarrow 466)($ e), at $m / z 480(744 \rightarrow 480)($ h), and their MS 4 spectra of the ions at $m / z 401(726 \rightarrow 462 \rightarrow 401)($ c), at $m / z 405(732 \rightarrow 466 \rightarrow 405)(\mathbf{f})$, and at $m / z$ $419(744 \rightarrow 480 \rightarrow 419)(\mathbf{i})$

Further dissociation of the ion at $m / z 462(726 \rightarrow 462)$ (Figure $1 \mathrm{~b}$ ) from $\mathrm{m} / \mathrm{z} 726$ gives rise to the prominent ion at $\mathrm{m} / \mathrm{z} 196$, arising from loss of the 1-O-alk-1'-enyl residue at $s n-1$ as an alcohol (loss of $\mathrm{HO}-\mathrm{CH}=\mathrm{CH}-$ $\left.\mathrm{C}_{16} \mathrm{H}_{31}\right)$. The prominent ion at $\mathrm{m} / \mathrm{z} 401$ arises from further loss of the ethanolamine $\left(\mathrm{HOCH}_{2} \mathrm{CH}_{2} \mathrm{NH}_{2}\right)$ group, and the ions at $\mathrm{m} / \mathrm{z} 140$ and 153 probably represent a phosphoethanolamine and a 2-hydroxy1,3-cyclophosphoric anions, respectively (Scheme 1A). These ions signify that the compound is a GPEtn [10]. The spectrum also contains the ion at $\mathrm{m} / \mathrm{z} 265$, corresponding to an ${ }^{-} \mathrm{O}-\mathrm{CH}=\mathrm{CH}-\mathrm{C}_{16} \mathrm{H}_{31}$ ion, consistent with the presence of the 1-O-alk-1'-enyl residue at sn-1. In contrast, the IT MS ${ }^{3}$ spectrum of the ion at $m / z 466$ (732 $\rightarrow$ 466) (Figure 1e) derived from a18:0/18:0-GPEtn is dominated by the ion at $\mathrm{m} / \mathrm{z} 405$, arising from loss of the ethanolamine residue (Scheme 1B). The spectrum is also featured by the phosphoethanolamine anion at $\mathrm{m} / \mathrm{z}$ 140 , along with the ion at $m / z 196$, arising from loss of the 1-O-octadecanyl residue as an alcohol (loss of
$\left.\mathrm{C}_{18} \mathrm{H}_{37} \mathrm{OH}\right)$. However, the ion at $\mathrm{m} / \mathrm{z} 196$ is of low abundance, consistent with the presence of the weak ion at $m / z 269$. The results may suggest that the cleavage of the 1-O-alkyl residue to yield an alkoxide ion or to the ion at $m / z 196$ by loss of an alcohol is a less facile process than cleavage of the 1-O-alk-1'-enyl residue that results in the formation of the alk-1'-enoxide ion and ion at $\mathrm{m} / \mathrm{z} 196$ seen for a plasmalogen GPEtn. The substantial differences between the two spectra arising from a plasmenylethanolamine (Figure 1b) and a plasmanylethanolamine (Figure 1e) demonstrated that multiplestage tandem mass spectrometry employing $\mathrm{MS}^{3}$ is readily applicable in their structural identification.

The MS $^{3}$ spectra of $m / z 462(726 \rightarrow 462)$ (Figure $\left.1 b\right)$ and of $m / z 466(732 \rightarrow 466)$ (Figure 1e) are identical to $\mathrm{MS}^{2}$ spectra of the $[\mathrm{M}-\mathrm{H}]^{-}$ions of 1-O-octadec-1'enyl-2-lyso-sn-glycero-3-phosphoethanolamine ( $p 18: 1 /$ 0-GPEtn) at $m / z 462$ and of 1-O-octadecanyl-2-lyso-snglycero-3-phosphoethanolamine (a18:0/0-GPEtn) at $\mathrm{m} / \mathrm{z}$ 466 (data not shown), respectively. These results are 


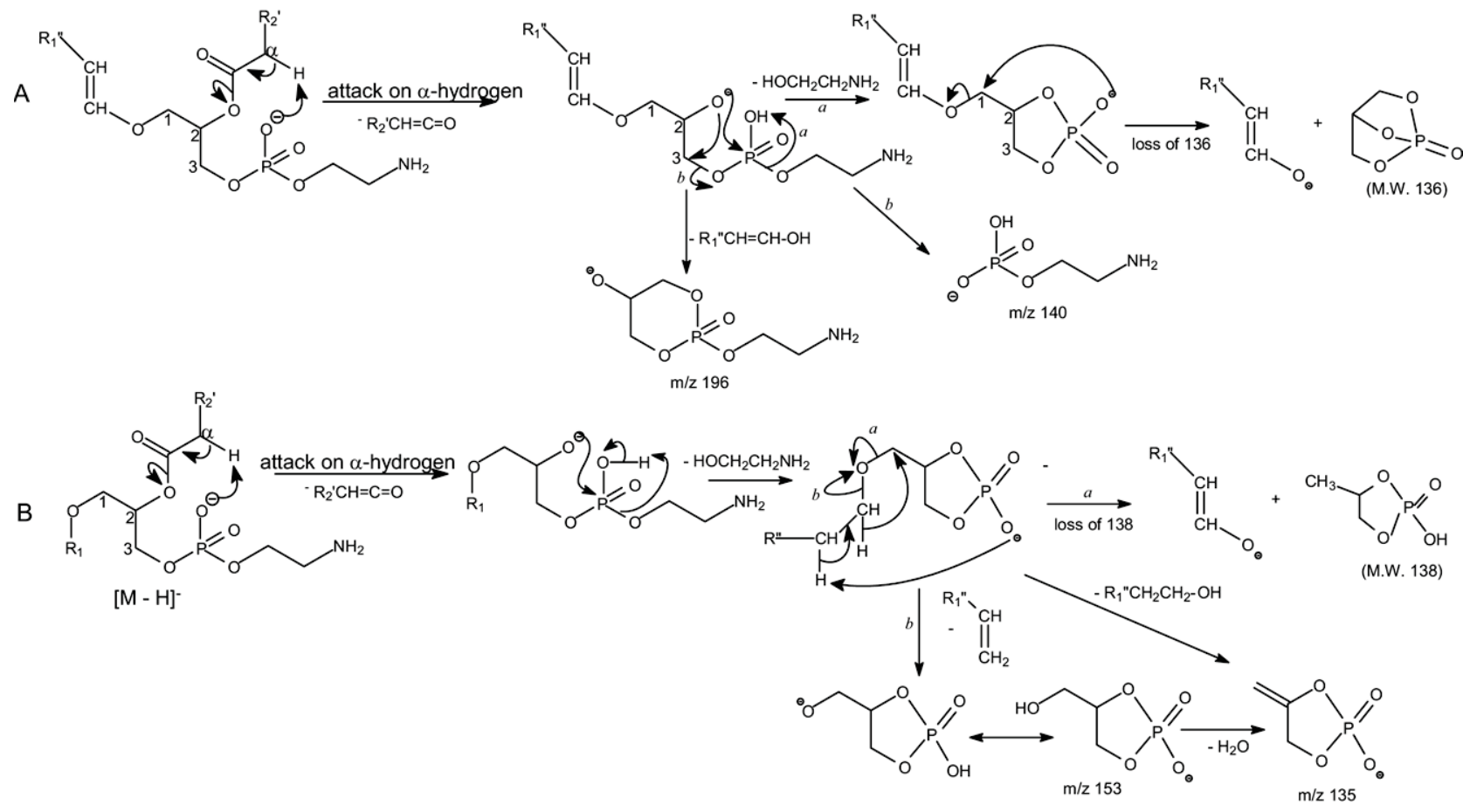

Scheme 1. The fragmentation pathways proposed for the $[\mathrm{M}-\mathrm{H}]$ ions of $(\mathbf{A})$ plasmenylethanolamine and (B) plasmanylethanolamine.

consistent with the notion that the ions seen at $\mathrm{m} / \mathrm{z} 462$ in Figure 1a and at $m / z 466$ in Figure 1d arise from $\mathrm{m} / \mathrm{z}$ 726 and 732, respectively, by primary losses of the 18:1and 18:0-fatty acyl substituents at $s n-2$ as a ketene, respectively.

The LIT MS ${ }^{2}$ spectrum of the $[\mathrm{M}-\mathrm{H}]^{-}$ion from a diacyl-GPEtn is readily applicable for structural assignment. For example, the $\mathrm{MS}^{2}$ spectrum of the $[\mathrm{M}-\mathrm{H}]^{-}$ ion of 18:0/18:1-GPEtn at $\mathrm{m} / \mathrm{z} 744$ (Figure 1g) contains the ions at $\mathrm{m} / \mathrm{z} 480$ and 478 arising from losses of the 18:1-fatty acyl ketene at $s n-2$ and the 18:0-fatty acyl ketene at $s n-1$, respectively, along with the ions at $m / z 281$ and 283 , corresponding to 18:1- and 18:0-carboxylate anions, respectively [10]. The $\mathrm{MS}^{3}$ spectrum of the ion at $\mathrm{m} / \mathrm{z}$ 480 (744 $\rightarrow 480$, Figure $1 \mathrm{~h}$ ) is dominated by the carboxylate anion at $\mathrm{m} / \mathrm{z} 283$, and the spectrum is readily distinguishable from those seen in Figure $1 \mathrm{~b}$ and e. The distinction among the $\mathrm{MS}^{3}$ spectra (Figure $1 \mathrm{~b}, \mathrm{e}, \mathrm{h}$ ) of the analogous ions (i.e., the $\left[\mathrm{M}-\mathrm{H}-\mathrm{R}_{2}{ }_{2} \mathrm{CH}=\mathrm{C}=\mathrm{O}\right]^{-}$ ions) arising from various subclasses of GPEtn demonstrated the utility of multiple-stage tandem mass spectrometry employing $\mathrm{MS}^{3}$ in the differentiation of various GPEtn subclasses.

The IT MS ${ }^{4}$ spectrum of the ion at $m / z 401(726 \rightarrow$ $462 \rightarrow 401$, Figure 1c), formed from $p 18: 1 / 18: 1-G P E t n$ by consecutive losses of the 18:1-fatty acyl ketene and the ethanolamine moieties is dominated by the ion at $m / z 265$, corresponding to an ${ }^{-} \mathrm{O}-\mathrm{CH}=\mathrm{CH}-\mathrm{C}_{16} \mathrm{H}_{31}$ ion, and the ion at $\mathrm{m} / \mathrm{z} 135$, arising from loss of a HO$\mathrm{CH}=\mathrm{CH}-\mathrm{C}_{16} \mathrm{H}_{33}(268 \mathrm{Da})$ residue is of low abundance. By contrast, the IT MS ${ }^{3}$ spectrum of the ion at $\mathrm{m} / \mathrm{z} 405$
(734 $\rightarrow 466 \rightarrow 405$, Figure 1f), originated from a18:0/18: 0 -GPEtn is dominated by the ion at $m / z 135$, arising from further loss of the 1-O-alkyl ether as an alcohol (loss of $\mathrm{HO}-\mathrm{C}_{18} \mathrm{H}_{37}$ ), along with the ion at $\mathrm{m} / \mathrm{z} 267$, probably representing an ${ }^{-} \mathrm{O}-\mathrm{CH}=\mathrm{CH}-\mathrm{C}_{16} \mathrm{H}_{31}$ anion arising from neutral loss of 138 (Scheme 1B). The optimal relative collision energy required to obtain the spectrum shown in Figure 1f is also significantly higher than that required to obtain the spectrum shown in Figure 1c (24\% versus $15 \%)$. The apparent differences in these two spectra (Figure 1c and f) are consistent with the notion that they arise from a plasmenylethanolamine and a plasmanylethanolamine, respectively. The MS $^{4}$ spectrum of the ion at $\mathrm{m} / \mathrm{z} 419(744 \rightarrow 480 \rightarrow 419$, Figure 1i) originated from $m / z 744$ by consecutive losses of the 18:1-fatty acyl ketene and the ethanolamine residues is also dominated by the 18:0-carboxylate anion ion at $\mathrm{m} / \mathrm{z} 283$, together with the ion at $\mathrm{m} / \mathrm{z} 153$ arising from further loss of the 18:0-fatty acyl ketene. This distinction among the $\mathrm{MS}^{4}$ spectra (Figure 1c, f, i) of the analogous ions (i.e., the $\left[\mathrm{M}-\mathrm{H}-\mathrm{R}_{2}{ }_{2} \mathrm{CH}=\right.$ $\left.\mathrm{C}=\mathrm{O}-\mathrm{HOCH}_{2} \mathrm{CH}_{2} \mathrm{NH}_{2}\right]^{-}$ions) arising from various subclasses of GPEtn demonstrated that multiple-stage mass spectrometry with subsequent application of $\mathrm{MS}^{\mathrm{n}}$ $(n=2,3,4)$ is readily applicable in the identification of various GPEtn subclasses. Because the product-ion spectra of the $\left[\mathrm{M}-\mathrm{H}-\mathrm{R}_{2}{ }_{2} \mathrm{CH}=\mathrm{C}=\mathrm{O}-\mathrm{HOCH}_{2} \mathrm{CH}_{2} \mathrm{NH}_{2}\right]^{-}$ ions, which lead to the structural distinction, do not involve the polar head group, the approaches may also be useful in the differentiation of various subclasses of GPLs (shown later). 


\section{Characterization of Plasmenyl and Plasmanyl Glycerophospholipids in the Lipid Extract from Leishmania major}

The major ions belong to the GPEtn class found in $L$. major were seen at $m / z 726$ and 728 [11]. The LIT MS $^{2}$ spectrum of the ion at $m / z 726$ (Figure 2a) is dominated by the 18:1-carboxylate anion at $m / z 279$, together with the ions at $m / z 464$ and 446, arising from losses of the 18:2-fatty acid substituent as an acid and as a ketene, respectively. The results indicated that the compound contains a 18:2-fatty acyl moiety. Further dissociation of the ion at $m / z 464(726 \rightarrow 464$, Figure $2 b)$ gives rise to the ion at $m / z$ 403, arising from further loss of the ethanolamine group, and the ion at $m / z 267$, representing a ${ }^{-} \mathrm{O}-\mathrm{CH}=\mathrm{CH}-\mathrm{C}_{16} \mathrm{H}_{33}$ anion. The spectrum also contains the feature ions for an GPEtn that were seen at $m / z$ 196, arising from loss of the $s n-1$ 1-O-alk-1'-enyl residue as an alcohol, and the ion at $m / z 140$, corresponding to a phosphoethanolamine anion. The profile of the spectrum is similar to that shown in Figure $1 b$, indicating that the compound is a $p 18: 0 / 18: 2-G P E t n$. The structural assignment is further supported by the $\mathrm{MS}^{4}$ spectrum of the ion at $m / z 403$ (Figure 2c), of which the profile is identical to that shown in Figure 1c. The LIT $\mathrm{MS}^{2}$ spectrum of the ion at $\mathrm{m} / \mathrm{z} 728$ (Figure $2 \mathrm{~d}$ ) is similar to that shown in Figure 2a and its $\mathrm{MS}^{3}$ spectrum of the ion at $m / z 464(728 \rightarrow 464)$ and $\mathrm{MS}^{4}$ spectrum of the ion at $m / z 403(728 \rightarrow 464 \rightarrow 403$ ) (data not shown) are also identical to Figure $2 b$ and $c$, respectively, indicating that the ion at $m / z 728$ represents a $p 18: 0 / 18: 1-G P E t n$. However, the spectrum (Figure $2 \mathrm{~d}$ ) also contains another set of the ions at $m / z 279$, corresponding to a 18:2-carboxylate anion and at $m / z 466$ and 405, arising from losses of the 18:2-fatty acyl substituent as a ketene and as an acid, respectively. The $\mathrm{MS}^{3}$ spectrum of the ion at $\mathrm{m} / \mathrm{z} 466$ $(728 \rightarrow 466)$ and the $\mathrm{MS}^{4}$ spectrum of the ion at $m / z 405$ $(728 \rightarrow 466 \rightarrow 405)$ (data not shown) are identical to those shown in Figure 1e and Figure 1f, respectively. The results suggested the ion at $m / z 728$ may also consist of an a18:0/18:2-GPEtn isomer.
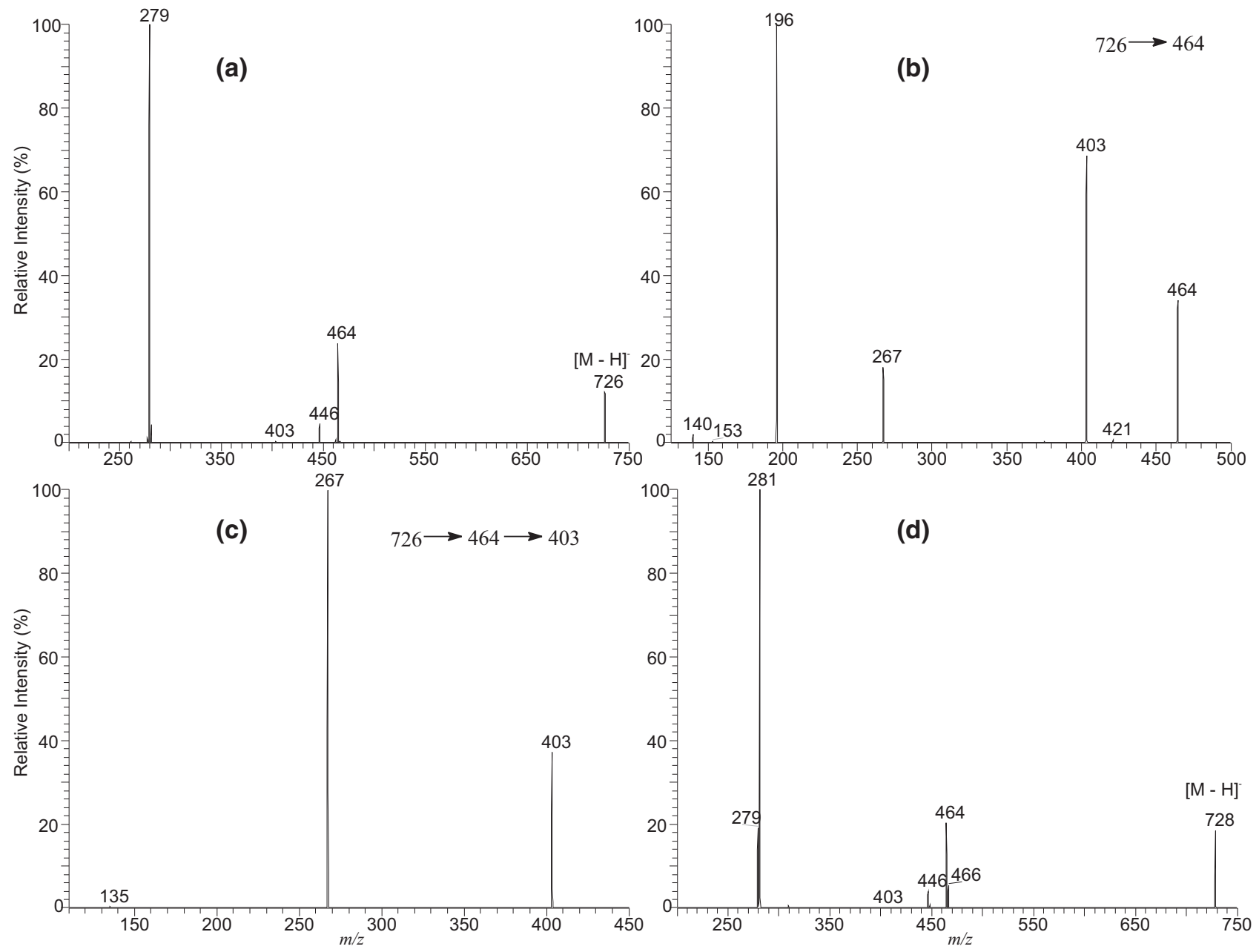

Figure 2. The LIT MS ${ }^{2}$ spectrum of the $[\mathrm{M}-\mathrm{H}]^{-}$ion of $p 18: 0 / 18: 2-\mathrm{GPE}$ tn at $m / z 726(\mathbf{a})$, its $\mathrm{MS}^{3}$ spectrum of the ion at $m / z 464(726 \rightarrow 464)(\mathbf{b})$, its MS ${ }^{4}$ spectrum of the ion at $m / z 403(726 \rightarrow 464 \rightarrow$ 403) (c), and the $\mathrm{MS}^{2}$ spectrum of the ion at $m / z 728$ (d), which consists of both a $p 18: 0 / 18: 1$-GPEtn structure and an a18:0/18:2-GPEtn structure. 
Several ions belong to the plasmanylinositol were observed. These ions were seen at $m / z 847,849$ and 933, corresponding to an a18:0/18:2-GPIns, a18:0/18:1GPIns and $a 24: 0 / 18: 1-G P I n s$, respectively [11]. The LIT $\mathrm{MS}^{2}$ spectrum of the ion at $\mathrm{m} / \mathrm{z} 849$ (Figure 3a) contains the ion at $m / z 567$ and 585, arising from losses of 18:1-fatty acid as an acid and as a ketene, respectively, along with the ion at $m / z 281$, representing a 18:1carboxylate anion. The prominent ion at $m / z 405$ (567162 ) arises from further loss of the inositol residue from $m / z 567$, and the ion at $m / z 241$ represents a inositol-1,2cyclic phosphate anion that is commonly seen for an GPIns [11, 12]. The above results indicated that the ion at $m / z 849$ is a GPIns consisting of a 18:1-fatty acyl residue, probably represents an a18:0/18:1-GPIns. The assignment of the $a 18: 0$ residue is supported by the LIT $\mathrm{MS}^{3}$ spectrum of the ion at $m / z 405(849 \rightarrow 405$, Figure $3 \mathrm{~b})$, which is nearly identical to that shown in Figure 1f.

In the same spectrum, an ion at $m / z 269$, probably representing a $\mathrm{C}_{16} \mathrm{H}_{33} \mathrm{CO}_{2}{ }^{-}$anion was also observed
(Figure $3 b$ ). This minor peak may indicate the presence of a 17:0/18:1-GPIns isomer, which yielded the ion at $\mathrm{m} / \mathrm{z} 405$ by primary losses of the 18:1-fatty acid substituent and the inositol head group, followed by loss of a 136 residue, probably a bicyclic glycerophosphate ester moiety [12, 13], to a 17:0-carboxylate anion at $m / z 269$ via the fragmentation process as seen for a diacyl-GPIns [12], a similar process that also yields the $\mathrm{MS}^{4}$ spectrum as shown in Figure 1i. The results are also consistent with the accurate mass measurement of the precursor ion with a LTQ-FT instrument, which gives a major ion at $m / z$ 849.5856, corresponding to $\mathrm{C}_{45} \mathrm{H}_{86} \mathrm{O}_{12} \mathrm{P}_{1}$ (calculated mass: 849.5857 ) that arises from the a18:0/ 18:1-GPIns structure, along with a minor peak at $\mathrm{m} / \mathrm{z}$ 849.5488, corresponding to $\mathrm{C}_{44} \mathrm{H}_{82} \mathrm{O}_{13} \mathrm{P}_{1}$ (calculated mass: 849.5493) arising from 17:0/18:1-GPIns.

The LIT MS ${ }^{2}$ spectrum of the ion at $m / z 847$ (Figure $3 d$ ) is similar to Figure 3a. The 18:2-fatty acid substituent at $s n-2$ is recognized by the ions at $m / z 585$ and 567, arising from losses of the 18:2-fatty acid as a ketene and
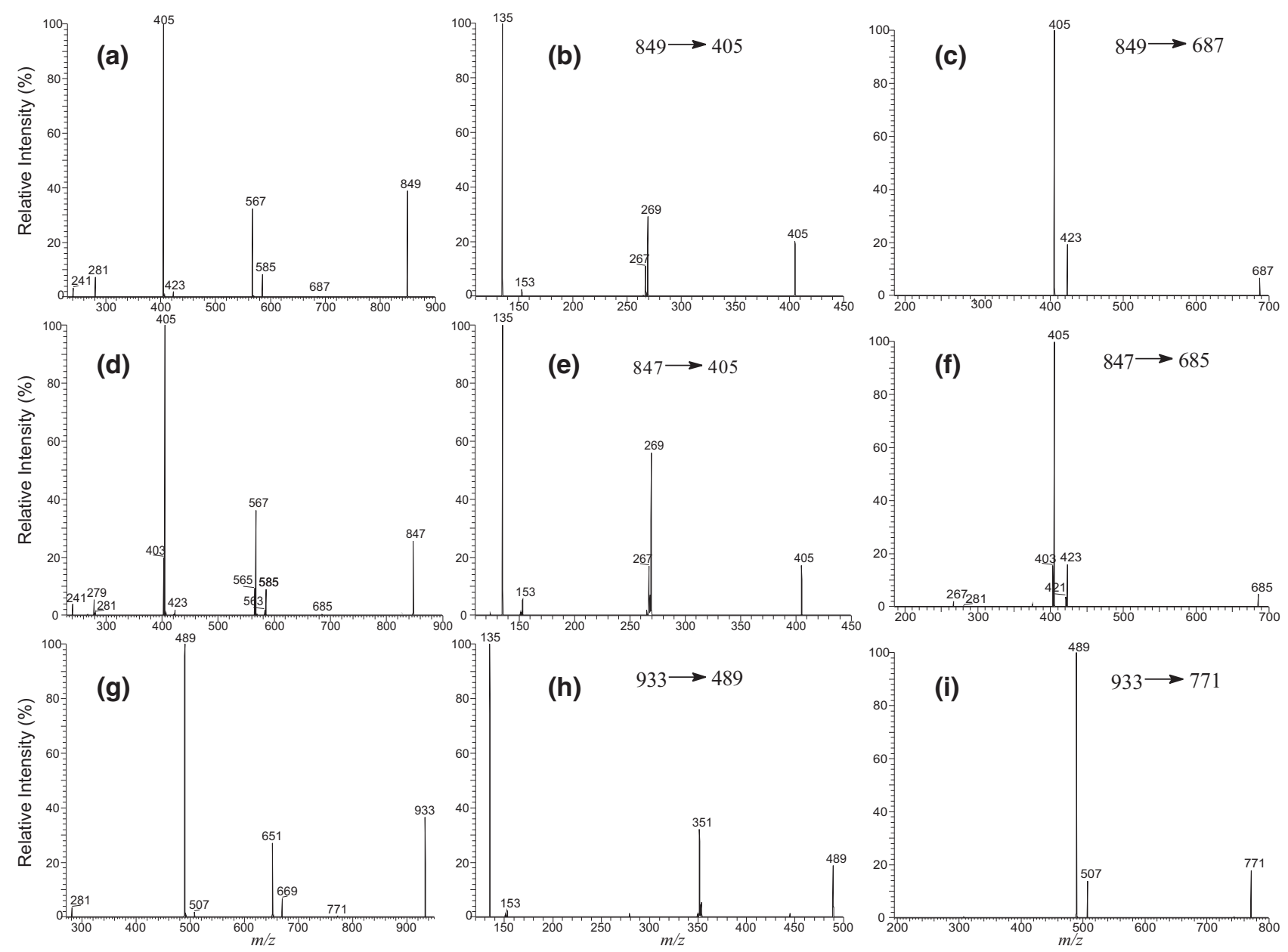

Figure 3. The LIT MS ${ }^{2}$ spectrum of the $[\mathrm{M}-\mathrm{H}]^{-}$ions of $a 18: 0 / 18: 1-\mathrm{GPIns}$ at $m / z 849$ (a), and its $\mathrm{MS}^{3}$ spectra of the ions at $m / z 405(849 \rightarrow 405)(\mathbf{b})$, and at $\mathrm{m} / z 687(849 \rightarrow 687)(\mathbf{c})$. The MS ${ }^{2}$ spectrum of the ion at $m / z 847$ (d), as well as its MS $^{3}$ of the ions at $m / z 405$ (e), and at $m / z 685$ (f) represents mainly an a18:0/18:2-GPIns structure, along with the minor 17:0/18:2-GPIns and p18:1/18:1-GPIns species; while the $\mathrm{MS}^{2}$ spectrum of the ion at $m / z 933(\mathrm{~g})$, and its $\mathrm{MS}^{3}$ spectra of the ions at $m / z 489(933 \rightarrow 489)$

$($ h), and at $m / z 771(933 \rightarrow 771)(\mathbf{i})$, represent an $a 24: 0 / 18: 1-G P I n s$ structure. 
as an acid, respectively, along with the ion at $m / z 279$; while the $\mathrm{MS}^{3}$ spectrum of the ion at $m / z 405$ (Figure 3e, $847 \rightarrow 405)$ is similar to Figure $1 \mathrm{f}$, confirming the $a 18: 0$ structure at $s n-1$. The observation of the ion at $m / z 269$ in Figure $3 e$, again, indicated the presence of a 17:0-fatty acid substituent at $s n-2$ arising from a 17:0/18:2-GPIns isomeric structure. The results are also consistent with accurate mass measurements (data not shown). The ion pairs at $m / z 583$ and 565 arising from $m / z 847$ by losses of a 18:1-fatty acid substituent as a ketene and as an acid, respectively, along with the ion at $m / z 281$, representing a 18:1-carboxylate anion (Figure 3d) signify that a 18:1-fatty acid moiety is also present. These ions are formed concurrently with the ion at $m / z 403$ ([565-162] and [583-180], deriving from further losses of the various inositol moieties from $\mathrm{m} / \mathrm{z} 583$ (i.e., loss of 180) and 565 (i.e., loss of 162). The $\mathrm{MS}^{3}$ spectrum of the ion at $m / z 403(847 \rightarrow 403$, data not shown) is identical to that shown in Figure 2c. These results suggest that the ion at $m / z 847$ may also consist of a 18:0/18:1-GPIns structure.

The LIT MS ${ }^{2}$ spectrum of the ion at $m / z 933$ (Figure $3 \mathrm{~g}$ ) is also similar to that of $m / z 849$ (Figure 3a) and the spectrum is dominated by the ion at $m / z 489$ (933 $\mathrm{C}_{17} \mathrm{H}_{33} \mathrm{CO}_{2} \mathrm{H}-162$ ), an ion analogous to $m / z 405$ as seen in Figure 3a. The presence of the 18:1-fatty acid moiety at $s n-2$ is seen by the ions at $m / z 651$ (933 $\left.\mathrm{C}_{17} \mathrm{H}_{33} \mathrm{CO}_{2} \mathrm{H}\right)$ and $669\left(933-\mathrm{C}_{16} \mathrm{H}_{31} \mathrm{CHC}=\mathrm{O}\right)$, along with the 18:1-carboxylate anion at $m / z 281$. The $\mathrm{MS}^{3}$ spectrum of the ion at $m / z 489(933 \rightarrow 489$, Figure $3 \mathrm{~h}$ ) contains the ion at $m / z$ 351, arising from loss of a 138 residue as described earlier and the profile of the spectrum is similar to that of Figure 1f, suggesting that the ion at $m / z 489$ is derived from a plasmanyl-GPL rather than from a plasmenyl-GPL. The results support that the ion at $m / z 933$ is indeed an $a 24: 0 / 18: 1-G P I n s$.

In the lipid extract obtained from African trypanosomes [9], an ion at $m / z 772$ was observed. The IT MS $^{2}$ spectrum of the ion at $m / z 772$ (Figure 4a) is dominated by the ion at $m / z 685$, probably corresponding to loss of a serine head group (loss as [serine $-\mathrm{H}_{2} \mathrm{O}$ ]) [14]. Further dissociation of the ion at $\mathrm{m} / \mathrm{z} 685$ gives rise to ions at $\mathrm{m} / \mathrm{z}$ 405 and 423 , by losses of 18:2-fatty acid substituent as an acid and as a ketene, respectively (Figure 4b, $772 \rightarrow$ 685). The spectrum also contains the ion at $m / z 279$, corresponding to a 18:2-carboxylate anion. The results indicate that the ion at $m / z 772$ is a glycerophosphoserine (GPSer) containing a 18:2-fatty acid substituent. The $\mathrm{MS}^{3}$ spectrum of the ion at $\mathrm{m} / \mathrm{z} 405$ (Figure $4 \mathrm{c}$, $772 \rightarrow 405)$ and the $\mathrm{MS}^{4}$ spectrum of the ion at $m / z 405$ $(772 \rightarrow 685 \rightarrow 405$, data not shown) are identical and the spectra contain the ions at $m / z 267$ and 135, indicating the presence of an $a 18: 0$ moiety at $s n-1$. The results suggest that the ion at $m / z 772$ is mainly an $a 18: 0 / 18: 2$ GPSer. However, the spectrum (Figure 4c) also contains a prominent ion at $m / z 269$, probably representing a 17:0-carboxylate anion, together with the ion at $m / z 153$, arising from loss of a 17:0-fatty acid moiety as a ketene as described earlier. These ions may indicate that the
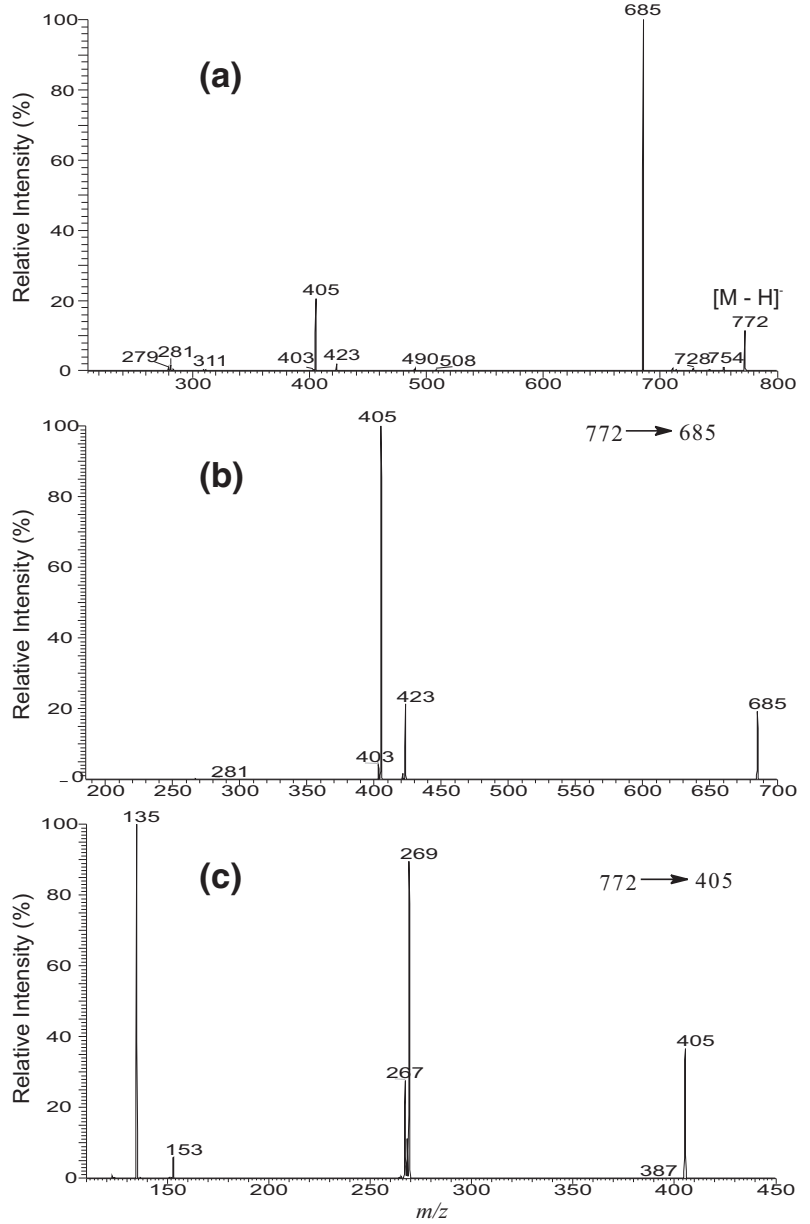

Figure 4. The LIT MS ${ }^{2}$ spectrum of the ion at $m / z 772\left(\right.$ a), its $\mathrm{MS}^{3}$ spectrum of the ion at $m / z 685(772 \rightarrow 685)\left(\right.$ b), and its $\mathrm{MS}^{4}$ spectrum of the ion at $m / z 405(772 \rightarrow 685 \rightarrow 405)($ c), from isobaric isomers of a18:0/18:2-GPSer, 17:0/18:2-GPSer, and p18:0/18:1GPSer.

ion at $m / z 772$ also consist of a 17:0/18:2-GPSer structure. The assignment of the minor 17:0/18:2-GPSer structure is also consistent with the results from highresolution mass measurement of the precursor ions, which give the major ion at $m / z 772.5508$ corresponding to a elemental composition of $\mathrm{C}_{42} \mathrm{H}_{79} \mathrm{O}_{9} \mathrm{~N}_{1} \mathrm{P}_{1}$ (calculated: 772.5492) from a18:0/18:2-GPSer, and a minor peak at $\mathrm{m} / \mathrm{z} 772.5120$ corresponding to a elemental composition of $\mathrm{C}_{41} \mathrm{H}_{75} \mathrm{O}_{10} \mathrm{~N}_{1} \mathrm{P}_{1}$ (Calculated: 772.5128) from 17:0/18:2-GPSer.

In Figure $4 a$, an ion at $m / z 403$ (685 - 282), arising from loss of a 18:1-fatty acid moiety is also observed. The presence of this ion is consistent with the observation of the ion at $m / z 281$, corresponding to a 18:1carbolylate anion, suggesting that a GPSer consisting of a 18:1-fatty acyl group may also exist. The $\mathrm{MS}^{3}$ spectrum of the ion at $m / z 403$ (data not shown) is identical to that shown in Figure 2c, indicating that a compound with $p 18: 0 / 18: 1-G P S e r$ structure may also exist.

The ion at $m / z 685$ (Figure $4 a$ ) deriving from loss of a serine group from an analogous GPSer ion at $m / z 772$ is 
equivalent to a deprotonated glycerophosphoric acid (GPA) anion [14]. Similarly, the ions at $m / z 687,685$, and 771 seen in the LIT MS ${ }^{2}$ spectra of the ions at $\mathrm{m} / \mathrm{z} 849$ (Figure 3a), 847 (Figure 3d), and at 933 (Figure 3g), respectively, are also equivalent to phosphatidic anions, arising from loss of an inositol group [12]. The $\mathrm{MS}^{3}$ spectra of the ions at $m / z 687$ ( $849 \rightarrow 687$, Figure 3c), 685 $(847 \rightarrow 685$, Figure $3 f)$, and 771 (933 $\rightarrow$ 771, Figure 3 i) are similar to that shown in $4 \mathrm{~b}$, and the $\mathrm{MS}^{4}$ spectra of the ions at $m / z 405(849 \rightarrow 687 \rightarrow 405), 405(847 \rightarrow$ $685 \rightarrow 405)$ and $489(933 \rightarrow 771 \rightarrow 489)$ (data not shown) are also identical to Figure $3 \mathrm{~b}, \mathrm{e}$, and $\mathrm{h}$, respectively. These results indicate that the above LIT multiple-stage mass spectrometric method with successive application of $\mathrm{MS}^{2}$ and $\mathrm{MS}^{3}$ should also be readily feasible for identification of plasmenyl- and plasmanyl-GPA molecules.

\section{Identification of Isobaric Plasmalogen Glycerophosphoethanolamine Structures in Bovine Brain Extract}

Several plasmalogen GPEtns that consist of multiple isomeric structures were seen in the lipid extract from bovine brain. For example, the LIT MS ${ }^{2}$ spectrum of the ion at $\mathrm{m} / \mathrm{z} 750$ (Figure 5a) contains the carboxylate anions at $m / z 331,305$, and 303, representing the 20:4-, 20:3-, and 22:4-fatty acyl substituents, respectively. The observation of the above ions is consistent with the presence of the ion pairs at $\mathrm{m} / \mathrm{z} 464 / 446,462 / 444$, and $436 / 418$ arising from losses of the 20:4-, 20:3-, and 22:4-fatty acyl substituents as a ketene and as an acid, respectively. The LIT MS ${ }^{3}$ spectrum of the ions at $\mathrm{m} / \mathrm{z}$ $462(750 \rightarrow 462)$, and at $\mathrm{m} / z 464(750 \rightarrow 464)$ (data not shown) are identical to Figure $1 \mathrm{~b}$ and Figure $2 \mathrm{~b}$, respectively, and the profile of the $\mathrm{MS}^{3}$ spectrum of the ion at $\mathrm{m} / \mathrm{z} 436$ (Figure $5 \mathrm{~b}$ ) is also similar, suggesting that the ion at $\mathrm{m} / \mathrm{z} 750$ consists of a major $p 18: 0 / 20: 4$-GPEtn molecule together with a minor $p 18: 1 / 20: 3-G P E t n$ and $p$ 16:0/22:4-GPEtn structures. These structural assignments were further supported by the $\mathrm{MS}^{4}$ spectra of the ions at $m / z 403(750 \rightarrow 464 \rightarrow 403$ ) (data not shown), 401 $(750 \rightarrow 462 \rightarrow 401)$ (data not shown), and at $\mathrm{m} / z 375$ (750 $\rightarrow 436 \rightarrow 375$ ) (Figure 5c). The former two spectra are identical to those shown in Figure $2 \mathrm{c}$ and Figure 1c, respectively; and the profile of Figure $5 \mathrm{c}$ is also similar to Figure 1c (or Figure 2c) consistent with the structural assignments.

\section{Conclusions}

We demonstrated multiple-stage mass spectrometry implementing $\mathrm{MS}^{3}$ or $\mathrm{MS}^{4}$ on the $[\mathrm{M}-\mathrm{H}]^{-}$ions of GPL that have undergone consecutive losses of the fatty acid substituent at $s n-2$ and the polar head group (i.e., the $\left[\mathrm{M}-\mathrm{H}-\mathrm{R}_{2} \mathrm{CO}_{2} \mathrm{H}-\text { polar head group }\right]^{-}$ions), are readily applicable in the differentiation of the plasmanyl-, plasmenyl- and diacyl-glycerophospholipid subclasses
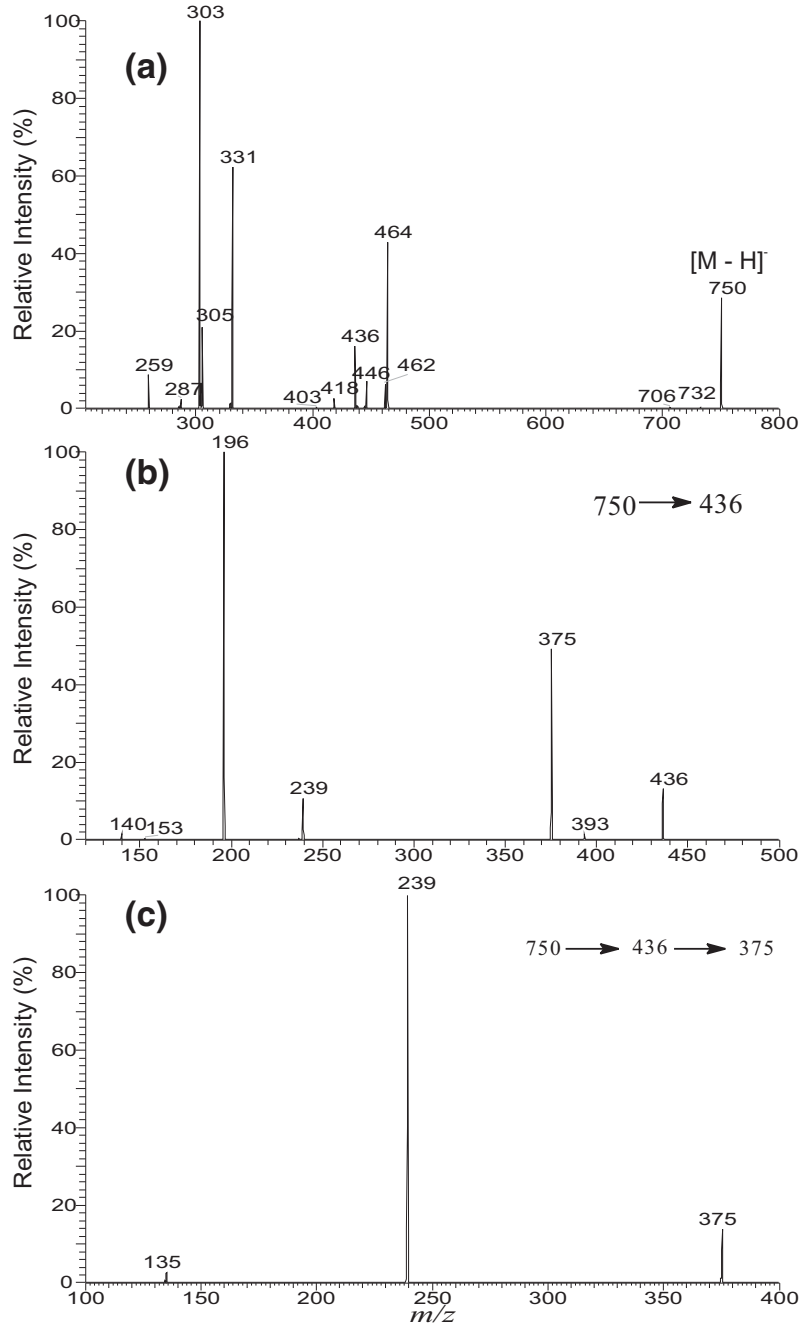

Figure 5. The LIT MS ${ }^{2}$ spectrum of the ion at $m / z 750(\mathbf{a})$, its $\mathrm{MS}^{3}$ spectrum of the ion at $\mathrm{m} / \mathrm{z} 436(750 \rightarrow 436)(\mathbf{b})$, and its $\mathrm{MS}^{4}$ spectrum of the ion at $m / z 375(750 \rightarrow 436 \rightarrow 375)($ c), from a major $p 18: 0 / 20: 4-G P E t n$ structure together with two minor $p 18: 1 / 20: 3$ GPEtn and $p 16: 0 / 22: 4-G P E t n$ isomers.

of GPLs, including GPEtn, GPIns, and GPSer. Thus, structures of GPL with isobaric isomers in mixtures can be unambiguously unveiled. Because the product-ion spectrum of the $\left[\mathrm{M}-\mathrm{H}-\mathrm{R}_{2} \mathrm{CO}_{2} \mathrm{H} \text { - polar head group }\right]^{-}$ ion that leads to the structural differentiation involves only the substituent at $s n-1$, this mass spectrometric approach should also be applicable for GPA and GPGro, of which the $\operatorname{MS}^{\mathrm{n}}(n=2,3)$ spectra also contain the similar precursor ions (i.e., the $[\mathrm{M}-\mathrm{H}-$ $\mathrm{R}_{2} \mathrm{CO}_{2} \mathrm{H}$ - polar head group] $]^{-}$ion) [15] that can be subjected to further dissociation.

\section{Acknowledgments}

Research at the Mass Spectrometry Resource of Washington University was supported by U.S. Public Health Service grants P41-RR-00954, R37-DK-34388, P60-DK-20579, P01-HL-57278, and P30-DK56341. The authors gratefully acknowledge Dr. Stephen M. Beverley and Dr. Kai Zhang of Washington University, St. Louis, 
and Dr. Jay D. Bangs and Dr. Shaheen S. Sutterwala of the University of Wisconsin, for providing the lipid samples.

\section{References}

1. Kayganich, K. A.; Murphy, R. C. Fast Atom Bombardment Tandem Mass Spectrometric Identification of Diacyl, Alkylacyl, and Alk-1Enylacyl Molecular Species of Glycerophosphoethanolamine in Human Polymorphonuclear Leukocytes. Anal. Chem. 1992, 64, 2965-2971.

2. Zemski-Berry, K. A.; Murphy, R. C. Electrospray Ionization Tandem Mass Spectrometry of Glycerophosphoethanolamine Plasmalogen Phospholipids. J. Am. Soc. Mass Spectrom. 2004, 15, 1499-1508.

3. Ramansdham, S; Hsu, F. F.; Bohrer, A.; Nowatzke, W.; Ma, Z., Turk, J. Electrospray Ionization Mass Spectrometric Analyses of Phospholipids from Rat and Human Pancreatic Islets and Subcellular Membranes: Comparison to Other Tissues and Implications for Membrane Fusion in Insulin Exocytosis. Biochemistry 1998, 37, 4553-4567.

4. Hsu, F. F.; Turk, J.; Thukkani, A. K.; Messner, M. C.; Wildsmith, K. R.; Ford, D. A. Characterization of Alkylacyl, Alk-1-Enylacyl, and Lyso Subclasses of Glycerophosphocholine by Tandem Quadrupole Mass Spectrometry with Electrospray Ionization. J. Mass Spectrom. 2003, 38, 752-763

5. Hsu, F. F.; Turk, J. Characterization of Phosphatidylethanolamine as a Lithiated Adduct by Triple Quadrupole Tandem Mass Spectrometry with Electrospray Ionization. J. Mass Spectrom. 2000, 35, 595-606.

6. Hsu, F. F.; Turk, J. Electrospray Ionization with Low-Energy Collisionally Activated Dissociation Tandem Mass Spectrometry of Complex Lipids: Structural Characterization and Mechanisms of Fragmentation. In Modern Methods for Lipid Analysis by Liquid Chromatography/Mass Spectrometry; Byrdwell, W. C., Ed.; AOCS Press: Champaign, IL, 2005; pp 61-178.
7. Pulfer, M.; Murphy, R. C. Electrospray Mass Spectrometry of Phospholipids. Mass Spectrom. Rev. 2003, 22, 332-364.

8. Hsu, F. F.; Turk, J. Zhang, K.; Beverley, S. M. Characterization of Inositol Phosphorylceramides from Leishmania major by Tandem Mass Spectrometry with Electrospray Ionization. J. Am. Soc. Mass Spectrom. 2007, $18,1591-1604$

9. Sutterwala, S. S.; Creswell, C. H.; Sanyal, S.; Menon, A. K.; Bangs, J. D. De Novo Sphingolipid Synthesis is Essential for Viability, but not for Transport of Glycosylphosphatidylinositol-Anchored Proteins, in African Trypanosomes. Eukaryotic Cell 2007, 6, 454-464.

10. Hsu, F. F.; Turk, J. Charge-Remote and Charge-Driven Fragmentation Processes in Diacyl Glycerophosphoethanolamine Upon Low-Energy Collisional Activation: A Mechanistic Proposal. J. Am. Soc. Mass Spectrom. 2000, 10, 892-899.

11. Zufferey, R.; Allen, S.; Barron, T.; Sullivan, D. R.; Denny, P. W.; Almeida, I. C.; Smith, D. F.; Turco, S. J.; Ferguson, M. A.; Beverle, S. M. Ether phospholipids and glycosylinositolphospholipids are not required for amastigote virulence or for inhibition of macrophage activation by Leishmania major. J. Biol. Chem. 2003, 278, 44708-44718.

12. Hsu, F. F.; Turk, J. Characterization of Phosphatidylinositol, Phosphatidylinositol-4-Phosphate, and Phosphatidylinositol-4,5-Bisphosphate by Electrospray Ionization Tandem Mass Spectrometry: A Mechanistic Study. J. Am. Soc. Mass Spectrom. 2000, 11, 986-999.

13. Hsu, F. F.; Turk, J. Charge-Driven Fragmentation Processes in Diacyl Glycerophosphatidic Acids Upon Low-Energy Collisional Activation. A Mechanistic Proposal. J. Am. Soc. Mass Spectrom. 2000, 11, 797-803.

14. Hsu, F. F.; Turk, J. Studies on Phosphatidylserine by Tandem Quadrupole and Multiple Stage Quadrupole Ion-Trap Mass Spectrometry with Electrospray Ionization: Structural Characterization and the Fragmentation Processes. J. Am. Soc. Mass Spectrom. 2005, 9, 1510-1522.

15. Hsu, F. F.; Turk, J. Studies on Phosphatidylglycerol with Triple Quadrupole Tandem Mass Spectrometry with Electrospray Ionization: Fragmentation Processes and Structural Characterization. J. Am. Soc. Mass Spectrom. 2001, 12, 1036-1043. 